\title{
Interactive comment on "Long-term relative decline in evapotranspiration with increasing runoff on fractional land surfaces" by Ren Wang et al.
}

\section{Rene Orth (Referee)}

rene.orth@bgc-jena.mpg.de

Received and published: 16 December 2020

Review of Wang et al. "Long-term relative decline in evapotranspiration with increasing runoff on fractional land surfaces"

This study derives land evapotranspiration with a machine learning approach applied to sub-daily meteorological station data from across the world. More decreasing than increasing trends are found across the Earth's land areas. The controls for these trends are determined and distinguished by jointly considering trends in evapotranspiration and precipitation minus evapotranspiration as a proxy for runoff.

Printer-friendly version

Discussion paper 
Recommendation: I think the paper requires major revisions.

The methodology and research question addressed in this paper are novel and relevant, making it a potentially good fit for HESS. Also the joint consideration of evapotranspiration and (proxy) runoff to interpret the reported trends and associate them with potential causes is an important contribution to the land surface science community. However, before the paper is suitable for publication in HESS, some critical shortcomings need to be addressed:

(1) I like that the authors validated their simulation results against observed streamflow and a reference ET product. However, the diagnosed agreement with these products is actually not very convincing, particularly in terms of the trends, as shown in Figure S8, and in the comparison between Figures $4 \mathrm{C}$ and S11. I think it is critical to understand these differences between the products, as otherwise I am missing convincing evidence that the data simulated here can be used to assess global ET and runoff trends.

(2) Adding to (1), it would be insightful to expand the cross-validation analysis from Figure 2 to validate the derived data also in terms of the trends observed at the independent cross-validation stations, as the final conclusions of this study are build on the trends rather than the short-term variability of the data.

(3) The comparison of ET and runoff trends between the CMIP5 scenario simuations and the machine learning-derived, historical simulations, does not really make sense as totally different time periods are considered to compute the respective trends, if I get this right?

(4) The description of the employed machine learning algorithm is not clear. The choice of artificial neural networks over other machine learning methods is not sufficiently motivated. Why is it more suitable than for example random forests in this context? Why 
not simply use FLUXCOM instead of deriving yet another estimate? Further, the setup of the ANN model is unclear, i.e. how the hyper-parameters are chosen (why exactly 2 hidden layers? why 500 epochs?), why different performance metrics (RMSE and MSE) are chosen, how the training is done, and how overfitting is prevented. I acknowledge that some of these choices are necessarily arbitrary, but this would be good to mention, including some tests on the relevance of these choices for the conclusions of the study.

(5) There are many small language errors (such as missing articles or wrong grammar) throughout the manuscript. The authors need to take special care of these when revising the manuscript.

I do not wish to remain anonymous - René Orth.

\section{Specific comments:}

lines 33 \& 36: Please explain what you mean with "offline"

lines 57, 72, 83, 123/124: You mention in these places different sets of variables which are (not?) used by the ANN algorithm, please clarify

line 69: How is this gap filling done? can it affect your results?

line 71: Why are you targeting daily resolution? To infer trends, monthly resolution might be sufficient?

lines 86-91: I do not fully understand this paragraph. Do the original and target station groups differ by the number of stations in some grid cells? If yes, do you average across multiple stations in one grid cell, or remove stations to only keep one (and which one?)?

line 101: Why do you emply top-of-atmosphere radiation instead of surface radiation which is what the vegetation is actually exposed to? 
lines 156/157: Where is the influence of the resistances shown, or how do you arrive to this conclusion?

line 160: How was the cross validation done? How/which stations or times have been chosen as independent validation data?

line 168: I would think that also SAV is water-limited?

lines 165, 169: Why are there different correlations given for $\mathrm{OSH}$ ?

line 184/185: What does 0.78 0.79 and 0.77 0.78 mean?

line 195: The reference for FLUXCOM would be Jung et al. 2019 which is also in the reference list, or did you actually use the MTE product from Jung et al. 2010?

line 220: I cannot see a cooling trend in northern Europe in Figure 3.

lines 226-228: Phrasing could be improved, the "when" is not needed here.

line 235: Not sure if I would see a "persistent long-term trend" anywhere here as the spatial patterns of trends vary quite a lot across time periods in Figure 5.

line 245: Which models did you consider in particular?

line 261: There is no "RCP8.5 climate model".

section 3.4: Very nice approach and analysis to infer potential causes of the observed trends.

Figure 1: What are the black empty bars which are superimposed on the colored bars?

Figure 3: How is the Sahara desert defined? Why are other deserts not excluded, too? What is the time period over which the trends are computed? How is the spatial interpolation between the station locations done?

Figure S10: This is cool, but where is the information coming from?

Printer-friendly version

Tables S1 and S2: Some of the variable names here need more explanation. And why

Discussion paper 
was only the u-component of the wind speed used?

References: Jung, M., et al. The FLUXCOM ensemble of global land-atmosphere HESSD energy fluxes, Scientific Data 6, 74 (2019). Jung, M., et al. Recent decline in the global land evapotranspiration trend due to limited moisture supply. Nature 467, 951-954 (2010).

Interactive comment on Hydrol. Earth Syst. Sci. Discuss., https://doi.org/10.5194/hess-2020590, 2020. 